\title{
Application Of High-Resolution Remote Sensing Images In Road Extraction
}

\author{
Huan Liu' ${ }^{1, a}$, Zhen Yan ${ }^{2, b}$ \\ ${ }^{1}$ Technology and Engineering Center for Space Utilization, Chinese Academy of Sciences, \\ Beijing, China \\ ${ }^{2}$ Technology and Engineering Center for Space Utilization, Chinese Academy of Sciences, \\ Beijing, China \\ aknowhuan@163.com \\ byanzhen@csu.ac.cn
}

Keywords: high-resolution remote sensing, road extraction, road feature, classification, difficulties Abstract. High-resolution remote sensing images includes high spectral resolution remote sensing images, high spatial resolution remote sensing images, high temporal resolution remote sensing images and so on. Based on the physical properties and functions, there are six road features: spectral feature, textural feature, geometric feature, topological feature, contextual feature and multi-scale feature, which have more information and research value in high-resolution remote sensing images. Starting from these features, the road extraction methods are analyzed and classified, then, the evaluation method and indicators for road extraction are introduced, the difficulties of road extraction from high-resolution remote sensing images are analyzed, and the trend of the research field is forecast in the end.

\section{Introduction}

High-resolution remote sensing technology is a rapidly developing subject of space science in recent years, which includes high spatial resolution, high spectral resolution, high temporal resolution and so on. Compared to low-resolution remote sensing images, it has larger coverage area, much larger information, better spectral continuity and better identification ability. So far, it has been widely applied in military and civilian fields, such as resource exploration, environmental monitoring, military reconnaissance, disaster monitoring and land planning, which promote the social and economic development to a great extent.

As one of common targets on the ground, road is not only a kind of important ground information, but a significant clue for extracting other ground targets. It has very bright prospect of extracting road correctly, likes updating of traffic database, updating of GIS database, urban planning, cartography......Currently, artificial cognition is the main method of road extraction from remote sensing images[1], which is hard to meet the production needs in speed, cost and efficiency. In order to promote production efficiency, it is necessary to develop automatic/semi-automatic road extraction means from high-resolution remote sensing images.

Starting from road features, the major methods and difficulties of road extraction from high-resolution remote sensing images are analyzed and concluded, and the trend of this field is forecast.

\section{Road Features}

The key of remote sensing application is how to extract image features[2]. $\mathrm{Wu}[3]$ classified road features into four categories: spectral feature, geometrical feature, topological feature and contextual feature. Then, the multi-scale feature was added by Miao[1] in 2014. As a conclusion, the road features are divided into six parts in this paper:

1) geometrical feature: road shows double edges parallel to each other and has little change in width; changes in curvature of the road should be within a certain range; the intersections of road 
usually look like a cross or "T".

2) spectral feature: generally, road regions have similar spectral character, while they have obvious spectral difference with neighboring regions.

3) textural feature: the road surface is generally uniform, and it has small gray variance and big consistency.

4) topological feature: commonly, road will not break off abruptly, oppositely, it usually forms road network.

5) contextual feature: information related to road, such as buildings and trees beside the road.

6) multi-scale feature: the same road has different width in different spatial resolution image, and different roads have multiple width in the same image.

Each feature has its own function in road extraction. For example, geometrical feature is closely related to the shape of road, spectral and textural features have close correlation with pavement materials and color, multi-scale feature can help improve the extraction result along with more cost.

Only with the combination of characteristics above, road can be extracted efficiently.

\section{The Methods And Research Status Of Road Extraction}

In the 21 st century, with the upgrading of remote sensing resolution, the study of road extraction has acquired unprecedented development. Aiming at diverse image data sources(such as high spectral images, SAR data, and LIDAR data), diverse image resolutions and diverse road types(such as rural road, urban road and expressway), researchers have proposed various methods to extract road.

Based on the degree of automation of road extraction, it was divided into semi-automatic extraction and automatic extraction by Luo[4], and he presented that the former is still the mainstream. According to the level of road knowledge used, Poullis and You[5] classified road detection methods into three categories: pixel-based; region-based; knowledge-based. In 2011, comprehensive summaries on the state-of-the-art road extraction methods were compiled by Das et al.[6].

In this paper, road extraction is classified into two categories: 1) based on single source; 2) based on multisource. The details are showed in Fig.1.

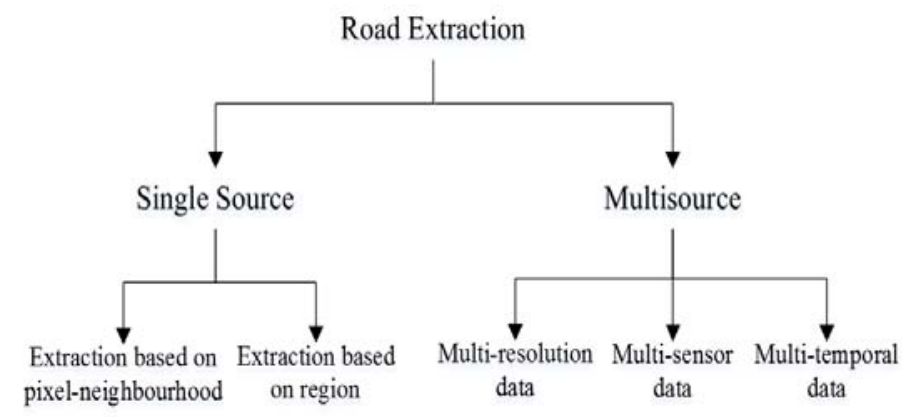

Fig.1. Classification in road extraction

\section{Road Extraction Based On Single Source}

This is a road detection and recognition process based on a single target image, which is the major research area on road extraction from remote sensing scene. It can be further divided into two parts: 1) road extraction based on pixel-neighbourhood; 2) road extraction based on region.

\section{Road extraction based on pixel-neighbourhood}

This extraction method refers that the road information is recognized through analyzing the difference between the target and background in certain neighbourhood of the target pixels. Some methods are summarized in this section.

1) Road extraction based on edge detection. The image edge is the place where there is pixel mutation. In this method, potential road points will be firstly detected by edge detection, then some necessary rules will be used to extract the road network. A novel system based on edge detection was proposed by Cem Unsalan and Beril Sirmacek[7] which consists of three main modules. In the 
first module, the road center line is extracted from the road edge information; in the second module, road shape and road width information are acquired via a novel ballon algorithm; then, former result is optimized with graph-theory-based road network formation(including edge information and neighbourhood information. The degree of automation and robustness are satisfactory while the accuracy is deficient.

2) Road extraction based on pixel reorganization. A variety of valuable information will be obtained by reorganizing image pixel, which make it become an effective tool in image processing. Structure tensor is such a matrix, compared with common gradient edge operators, it can describe geometric information more effectively, such as edge, shape and angular point of the target. An improved structure tensor was presented by Teng[8] which helped him extract road network successfully. In his method, the geometric feature, textural feature and spectral feature were included, and it is only suitable for high-resolution images because of the high requirement for pixel. Hessian matrix is also such a matrix which can describe local curvature of a function. Using a multi-scale Hessian matrix, Yoonsung Bae[9] smoothly shaped road profile based on which the complete road was extracted.

3) Road extraction based on template matching. In this method, target detection is generally completed via computing similarity between the template and the area covered by template. With $0.61 \mathrm{~m}$-resolution images, a total rectangle matching approach was presented by Zhu[10], in which the optimal rectangle represented the road. Based on the geometric feature and topological feature of road, one more road template matching approach was proposed by Koutaki[11]. The key of his approach is the detection of road intersection and the capture of templates (the templates were captured from the branch of intersections), and the drawback is the need for more manual intervention.

4) Road extraction based on snake model. Snake model, also called as active contour model, was firstly proposed by Kass in 1988[3], has been widely applied in image target extraction. The essence of the model is a deformable parametric curve and corresponding energy function, aiming at minimizing the energy function that is composed of internal force and image force[12]. When it comes to the road, the curvature and topological features are represented by the internal force, while the gradient and textural features are represented by the image force. On the base of these cognition, some modified snake models, such as quadratic snake[13] and network snake[14], have been used to extract road successfully.

\section{Road extraction based on region}

Generally speaking, road network is composed of a series of pixel regions with the same or similar properties, on the base of which, the target image is firstly segmented into road regions and background regions via certain suitable image segmentation[15] method, then the road network is extracted based on the road regions by means of some rules.

1) Road extraction based on multi-feature fusion. Spectral feature and geometric feature are the most widely used among road features because of their perceptual intuition and relative simpleness. Specifically, spectral feature is commonly used in analyzing spectral consistency inside roads and spectral differences between roads and the places around; as to geometric feature, there are some quantitative indexes expressing roads such as area index and LFI index. The former is actually a threshold value, regions with area beyond which can be deemed as roads. The latter refers length-width ratio of target regions:

$$
L F I=L_{M E R} / W_{M E R}
$$

In Eq.1, MER represents the minimum bounding rectangle of interesting regions, $L_{M E R}$ and $W_{M E R}$ respectively represents the length and width of the rectangle. Combined with characters above, a method based on image segmentation was proposed by Miao[16], in which the target image was firstly segmented into road regions and background regions through edge filter and thresholding method, the road parts were then modified by using appropriate area index and LFI index, the road 
center line were finally extracted by certain method. The extraction result is satisfactory while it is fit for high-resolution images merely because of the use of spectral consistency. Similarly, Shi et al.[17] presented an integrated method for urban main-road centerline extraction, in which the road spectral characters, area index and LFI index played important roles. Likewise, a method based on locally excitatory globally inhibitory oscillator networks was put forward by Yuan et al.[18] which has a high degree of automation and performed well on simple roads.

2) Road extraction based on mathematical morphology. Geometric feature is mainly researched in this theory, and dilation and erosion are the basic operations of it. Brightness can be highlighted and dark spots in images will be reduced or eliminated by means of dilation operation, oppositely, bright spots will be reduced or eliminated by means of erosion operation. Aiming at the occlusion caused by signs or vehicles on the road, morphological reconstruction operation was used to smooth the target image and extract the road by Wang[19]. In his method, road signs and vehicles were filtered by morphological reconstruction operation, then road regions were split out via binary processing, based on which dilation operation was used again to fill the holes and erosion operation was used to eliminate noisy dots.

3) Road extraction based on cluster analysis. Cluster analysis derives from taxonomy, it requires classes to be divided are unknown, that is, it belongs to unsupervised learning. K-means and fuzzy $\mathrm{C}$-means are common clustering methods. Utilizing FCM in image segmentation, an automatic road extraction method based on fuzzy connectedness was proposed by $\mathrm{Fu}$ et al.[20]. With regard to more interference in aeronautic images, Zhao et al.[21] presented road extraction based on clustering segmentation in which K-means operation was used in segmentation. Interference caused by high resolution is reduced effectively in this method.

4) Road extraction based on classifier. Different from cluster analysis, the classes to be divided are given, therefore, it belongs to supervised learning. Neural networks and support vector machine are commonly-used tooles. Using pulse coupled neutral network, $\mathrm{Xu}$ [22] realized road extraction with road blocks split out(these blocks were training samples of pulse coupled neutral network). In order to improve segmentation result, support vector machine was used by Miao[1], which helped him reduce the impact of noise.

\section{Road Extraction Based On Multisource}

Compared to road extraction based on single source, this kind of road extraction combines more information such as multi-resolution data, multi-sensor data and multi-temporal data that can contribute to extracting results. However, workload is increased so the pros and cons should be weighed in a practical application.

1) Road extraction based on multi-resolution data. The detection effects can be improved by applying the multi-resolution images. More road details can be acquired by high-resolution images, but the effect of noise would be magnified. Meanwhile, the road feature can be abstracted further by low resolution images, although some detail would be lost, noise jamming can be filtrated to some extent. The road extraction combined multi-resolution remote sensing images with the second generation curvelet fusion technology was realized by Zhang[23]. First, spectral similarity matrix was calculated by spectral images of different resolution, the spatial information could be reflected to some extent, then panchromatic image and spectral similarity matrix were fused using curvelet, finally the road was extracted by Hough transform.

2) Road extraction based on multi-sensor data. As for the application of multi-sensor data, a road extraction method was proposed by Cao et al.[24] by using data fusion of multi-spectral images and microwave radar images. In this method, the velocity function was applied by level set and fast marching, it combined spectral and textural features of road with the backward scattering field of radar image and spatial autocorrelation. The road was sheltered easily by building and shadow, which can be remedied by the road information in the radar images, and the noise jamming the radar images can be reduced by the road information in the optical images, such as the confusion of water and road.

3) Road extraction based on multi-temporal data. As for the use of multi-temporal data, a novel 
method was proposed by Vinay Pandit[25], in which the seed points were provided by the vehicle detection in multi-temporal images, then the road was traced by fourier description operator.

\section{The Evaluation Indexes For Road Extraction}

Effective evaluation criteria should be established in order to evaluate the effect of road extraction. Completeness, accuracy and quality are common in this filed.

$$
\begin{aligned}
& \text { Completeness }=\frac{l_{T E}}{l_{T E}+l_{E X}} . \\
& \text { Aceuracy }=\frac{l_{T E}}{l_{T E}+l_{E E}} . \\
& \text { Qualtiy }=\frac{l_{T E}}{l_{T E}+l_{E E}+l_{E X}} .
\end{aligned}
$$

Where $l_{T P}$ is the length of correct road extracted, $l_{F P}$ is the length of false road extracted, $l_{F N}$ is the length of missing road.

\section{Difficulties And Developing Trend Of Road Extraction}

Although research on road extraction from high-resolution remote sensing images has been going on for decades, it is still an international problem by now. There is not a method that can meet both extracting effect and universality. Some methods may have good extraction effect on a certain kind of road, but for other roads, it could hardly be satisfactory. Just as Fig. 2 shows, at least three factors contribute to this problem: 1) complexity of remote sensing scenes. 2) complexity of road itself. 3) limitations of algorithms. Remote sensing images provide a large number of surface features, what's more, with the increase of spatial resolution and spectral resolution, the spatial information become more complicated, and the spectrum aliasing become more serious, thus the disturbance of other features(building, trees, shadow) would become more obvious. Besides, the road is a very complex target, road especially urban road often presents complexity and diversity in images, such as the variety of road width and shape. Moreover, many methods on road extraction such as template matching method have limitation of their own, which will limit the universality of methods on road extraction to some extent.

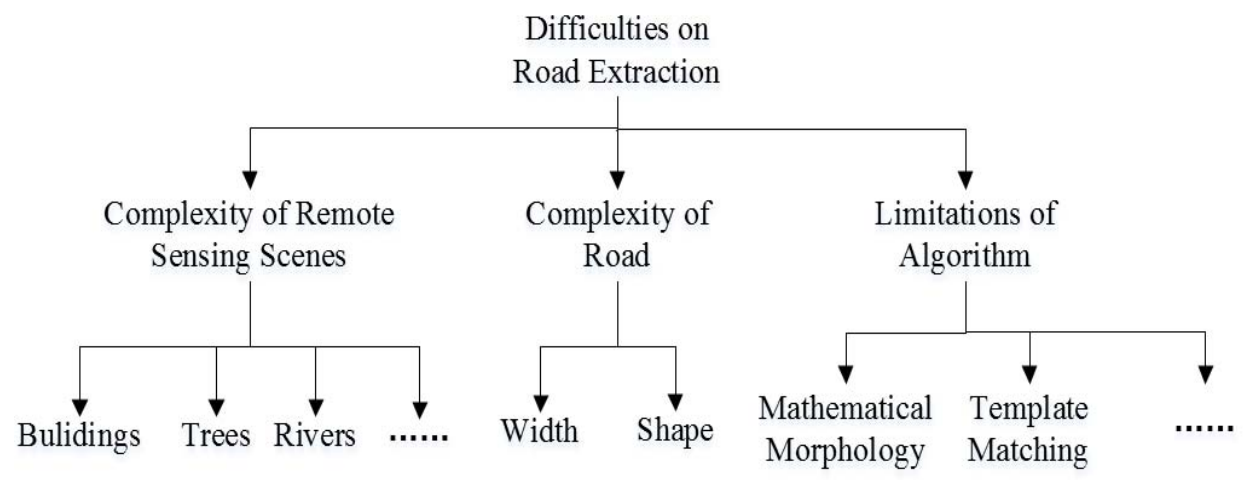

Fig.2. Difficulties in road extraction

It can be predicted that it is not realistic to realize automatic road extraction with satisfying universality and performance in a short future. The research of road extraction would continue concentrating on certain roads, certain resolutions and certain road types in coming decade. It is very hard to amend the error appeared in the process of road extraction only by characteristics of the road itself, thus more and more research would begin to utilize multisource data when high 
accuracy is required. Besides, there will be some research on the basic algorithms in image processing such as edge detection algorithm[26] improving the level of automation and universality of road extraction.

\section{Conclusions}

High-resolution remote sensing technology has become a main research field in space science. As one of important ground objects, the methods of road extraction from high-resolution images have received wide attention. Various characteristics of the road, such as geometric feature, spectral feature, textural feature and topological feature, become increasingly prominent in high-resolution remote sensing images, these characteristics provide effective basis for road extraction. According to the difference of information sources, the road extraction can be divided into two broad categories: road extraction based on single source and road extraction based on multisource, and the former is still the main research direction. Because of the complexity of high-resolution remote sensing scenes, the complexity of road itself and the limitations of algorithms, road extraction from high-resolution remote sensing images is still in the stage of development, with deficient automation and universality. In order to improve this state, some basic algorithms in image processing will be utilized more. In short, future research on road extraction from high-resolution remote sensing images will focus on improving the level of automation and universality, with the assurance of extracting quality.

\section{References}

[1] Zelang Miao. Study on Road Extraction From VHR Satellite Images Using Multiple Features[D]: [Master's Dissertation]. Xuzhou: College of Environment and Spatial in China University of Mining and Technology, 2014, In Chinese.

[2] Wenzhong Shi, Changqing Zhu, Yu Wang. Acta Geodaetica et Cartographica Sinica, 2001, 30(3): 257-262.

[3] Liang Wu, Y.A. Hu. ACTA AUTOMATICA SINICA, 2010, 36(7): 912-922.

[4] Zhaotuo Luo. Analysis and Research of Road Extraction From High Resolution Remote Sensing Images[D]: [Master's Dissertation]. Shanghai: College of Information and Electrical Engineering in Shanghai Jiao Tong University, 2008, In Chinese.

[5] C.Poullis, and S. You. ISPRS Journal of Photogrammetry and Remote Sensing. 2010, 65(2): 165-181.

[6] S.Das, T.T. Mirnalinee, and K. Varghese. IEEE Transactions on Geoscience and Remote Sensing, 2011, 49(10): 3906-3931.

[7] Cem Unsalan and Beril Sirmacek. IEEE Transactions on Geoscience and Remote Sensing, 2012, 50(11): 4441-4453.

[8] Xinpeng Teng. Research on Road Extraction from Remote Sensing Image[D]: [Ph.D. Dissertation]. Zhenjiang: Jiangsu University, 2014, In Chinese.

[9] Yoonsung Bae, Won-Hee Lee, Yunjun Choi, et al. IEEE Geoscience And Remote Sensing Letters, 2015, 12(9): 1858-1862.

[10] C.Q. Zhu, Y. Yang, Q.S. Wang, et al. Total rectangle matching approach for road extraction from high-resolution remote sensing images[A]. In: Proc. SPIE 6419, Geoinformatics 2006: Remotely Sensed Data and Information[C], 2006. 
[11] G Koutaki, K Uchimura. Automatic road extraction based on cross detection in suburb[A]. In: Proceedings of the Conference on Computational Imaging II. San Jose[C]. USA: SPIE, 2004: 337-344.

[12] Tianqing Li, Yi Zhang, Zhi Liu, et al. An Overview on Snakes Models[J]. Computer Engineering. 2005, 31(9).

[13] R Marikhu, M.N. Dailey, S Makhanov, K Honda. A family of quadratic snakes for road extraction. In: Proceedings of the 8th Asia Conference on Computer Vision. Tokyo, Japan: Spinger, 2007: 85-94.

[14] M Butenuth. Photogrammetrie Fernerkundung Geoinformation, 2007, 36(3): 1-6.

[15] Rafael C. Gonzalez, Richard E. Woods. Digital Image Processing[M]: Third Edition. Qiuqi Ruan, Yuzhi Ruan, ei al translate. Beijing: Publishing House of Electronics Industry, 2014.

[16] Zelang Miao, Wenzhong Shi, et al. IEEE Transactions on Geoscience and Remote Sensing, 2013, 10(3): 583 - 587.

[17] Wenzhong Shi, Zelang Miao, Johan Debayle. IEEE Transactions on Geoscience and Remote Sensing, 2014, 52(6): 3359-3372.

[18] Jiangye Yuan, DeLiang Wang, et al. IEEE Transactions on Geoscience and Remote Sensing, 2011, 49(11): 4528-4538.

[19] Ximing Wang. The Research and Application of Road Extraction from Remote Sensing Image[D]: [Master's Dissertation]. Beijing: Tsinghua University, 2011, In Chinese.

[20] Xiyou Fu, Fengli Fang, Guojun Wang, et al. Journal of Computer Applications, 2015, 35(2): 523-527.

[21] Yali Zhao, Yingmei Wei, Songyang Lao. Journal of System Simulation, 2014, 26(9): 2198-2202.

[22] Lili Xu. Study of The Road Semi-automatic Extraction Method from Satellite Image[D]: [Master's Dissertation]. Shenyang: Shenyang Aerospace University, 2010, In Chinese.

[23] Ye Zhang, Yijia Liu, and Junping Zhang. Road extraction from multi-source remote sensing images based on 2nd generation curvelet fusion[A]. In: Proceedings of Fifth International Conference on Natural Computation[C]. 2009: 247-251.

[24] Guangzhen Cao, Yaqiu Jin. Journal of Electronics and Information Technology, 2007, 29(6): 1464-1470, In Chinese.

[25] V Pandit, S Gupta, and K.S. Rajan. IEEE International Geoscience and Remote Sensing Symposium, 2009, 5: 272-275.

[26] Xiaofeng Li, Chi Zhang, Xin Pan, et al. Geomatics and Information Science of Wuhan University, 2010, 35(9): 1079-1081, In Chinese. 